Erwin J. O. Kompanje

Margo M. van Mol

Marjan D. Nijkamp

\section{'I just have admitted an interesting sepsis'. Do we dehumanize our patients?}

Received: 28 July 2015

Accepted: 5 August 2015

Published online: 14 August 2015

(C) Springer-Verlag Berlin Heidelberg and ESICM 2015

E. J. O. Kompanje ( ) M. M. van Mol

Department of Intensive Care Medicine, Erasmus MC University Medical Center, Rotterdam, The Netherlands

e-mail: e.j.o.kompanje@erasmusmc.nl

\section{E. J. O. Kompanje}

Department of Ethics and Philosophy of Medicine, Erasmus MC University Medical Center, Rotterdam, The Netherlands

M. D. Nijkamp

Faculty of Psychology and Educational Sciences, Open University, Heerlen, The Netherlands

'I just have admitted an interesting sepsis'; 'Do you have the lactate of that oesophagectomy on ICU-2?'; 'I asked the neurosurgeon to take a look at that bad-looking subarachnoid haemorrhage in box three'. These are three examples that the first author overheard physicians saying to each other when referring to patients in the intensive care unit (ICU). They were talking about $\mathrm{Mr} \mathrm{B}$., a 63-year-old male taxi driver ('the interesting sepsis'); Mrs C., a 69-year-old frail widowed woman ('the oesophagectomy') and Mrs D., a 49-year-old mother of three ('the bad-looking subarachnoid haemorrhage'). We wondered, would the young doctors also talk about 'an interesting sepsis', 'an oesophagectomy' or 'a bad looking subarachnoid haemorrhage' if it concerned their father, mother, sister, colleague or spouse? Probably not; that would be seen as inappropriate. It would be less empathic. But why do we so often talk about patients as if they were just diseases, operation techniques or organs? Why do we depersonalize or dehumanize them?

Another example: An 18-year-old girl was run over by a car when the driver failed to stop at a red traffic light. She was admitted to the hospital with severe traumatic brain injury. Her pupils were fixed, Glasgow Coma Scale 3 . One of us saw a senior intensivist, resident and nurse entering the family room to bring the news to the parents that their daughter was going to die. One hour later the intensivist and the resident were seen laughing, making jokes and enjoying lunch in the hospital restaurant. Were they indifferent to the immense catastrophe that the parents just had to deal with?

Every experienced health care provider recognizes these examples. Over time, most health care providers become desensitized to the physical and emotional pain, the distress, anxieties, fears and death of their patients, but usually retain the ability to know when a patient is in pain or distress. The dulling of their empathic sense is, however, essential for practising medicine.

A region in our frontal lobes, the medial prefrontal cortex, ensures that we can make inferences about the state of mind of others. When you think about how you can please someone, you use your medial prefrontal cortex. The intensivist speaking to the parents of the dying girl did not really feel the parents' pain, but he calmly inferred their pain. When people are unlike us, or more distant from our personal social networks (as patients are), they become less likely to engage our medial prefrontal cortex, making us more indifferent about the pain and suffering of others. That does not make bad health care providers. We do not want the health care providers to be suffering with the patients, but we want them to know that they suffer. Beside this, we should respond to the patients and their relatives with empathy, compassion and sensitivity. We must do more than simply 'know' they are suffering, but we must also alleviate that suffering to the best of our ability and, when we cannot 
alleviate their suffering, we should be able to be there as a witness and provide support through our presence.

Health care providers who are speaking about patients in terms of diseases, organs or operation techniques do so because it is practical. This is easier to remember in communication about the patient than using their real names. Because our primary interest lays in the disease, the failing organ, the pathophysiological puzzle and not in the person behind this, it is easier to communicate with colleagues in terms like 'the interesting sepsis' or the 'bad-looking subarachnoid haemorrhage'. Furthermore, the patients do not engage our medial prefrontal cortex, because of social-economic differences; conditions resulting from lifestyle choices that are not ours and because they, most importantly, are very distant from our social network. Finally, disease, pain, suffering and death are a daily part of the work in the ICU. The health care providers expect it and are not shocked or surprised to see it 24/7, 365 days a year. Through the 'dehumanization' of our patients we can deal with this.

An additional explanation can be found in the theory of cognitive dissociation. According to Festinger, a person cannot hold two cognitions that are inconsistent with one another; he will experience the tension of an aversive motivational state. This is a pressure that he will seek to remove, among other ways, by altering one of the two dissonant cognitions. How do health care providers reduce this tension? By changing his/her attitudes so that they are in line with our behaviour ('dehumanizing' patients so the health care providers do not feel the pain and suffering). Failing to do so may lead to burnout, compassion fatigue or even post-traumatic stress disorder.

So, yes, we dehumanize patients. But this is inevitable, adaptive and even morally and psychologically acceptable. As long as health care providers know that their patients are suffering and in pain, they do not have to feel it.

\section{Compliance with ethical standards}

Conflicts of interest The authors have no conflict of interest to declare. 